\title{
Video Article \\ Immunofluorescent Detection of Two Thymidine Analogues (CldU and IdU) in Primary Tissue
}

\author{
Alex H. Tuttle ${ }^{{ }^{1}}$, Matthew M. Rankin ${ }^{{ }^{1}}$, Monica Teta ${ }^{1}$, Daniel J. Sartori ${ }^{1}$, Geneva M. Stein ${ }^{1}$, Gina J. Kim ${ }^{1}$, Cristina Virgilio ${ }^{1}$, Anne Granger ${ }^{1}$, Di Zhou ${ }^{1}$, \\ Simon H. Long ${ }^{1}$, Alisa B. Schiffman ${ }^{1}$, Jake A. Kushner ${ }^{1}$ \\ ${ }^{1}$ Division of Endocrinology and Diabetes, Children's Hospital of Philadelphia, Institute of Diabetes Obesity and Metabolism, Institute for Regenerative Medicine, \\ Department of Pediatrics, University of Pennsylvania-School of Medicine \\ *These authors contributed equally
}

Correspondence to: Jake A. Kushner at kushnerj@mail.med.upenn.edu

URL: https://www.jove.com/video/2166

DOI: doi:10.3791/2166

Keywords: Immunology, Issue 46, CldU, IDU, Thymidine Analogue, immunofluorescence, Lineage-Tracing, Mouse, Cell Division, Replication, Turnover, Stem Cell

Date Published: 12/7/2010

Citation: Tuttle, A.H., Rankin, M.M., Teta, M., Sartori, D.J., Stein, G.M., Kim, G.J., Virgilio, C., Granger, A., Zhou, D., Long, S.H., Schiffman, A.B., Kushner, J.A. Immunofluorescent Detection of Two Thymidine Analogues (CldU and IdU) in Primary Tissue. J. Vis. Exp. (46), e2166, doi:10.3791/2166 (2010).

\section{Abstract}

Accurate measurement of cell division is a fundamental challenge in experimental biology that becomes increasingly complex when slowly dividing cells are analyzed. Established methods to detect cell division include direct visualization by continuous microscopy in cell culture, dilution of vital dyes such as carboxyfluorescein di-aetate succinimidyl ester (CFSE), immuno-detection of mitogenic antigens such as ki67 or PCNA, and thymidine analogues. Thymidine analogues can be detected by a variety of methods including radio-detection for tritiated thymidine, immuno-detection for bromo-deoxyuridine (BrdU), chloro-deoxyuridine (CldU) and iodo-deoxyuridine (IdU), and chemical detection for ethinyldeoxyuridine (EdU). We have derived a strategy to detect sequential incorporation of different thymidine analogues (CIdU and IdU) into tissues of adult mice. Our method allows investigators to accurately quantify two successive rounds of cell division. By optimizing immunostaining protocols our approach can detect very low dose thymidine analogues administered via the drinking water, safe to administer to mice for prolonged periods of time. Consequently, our technique can be used to detect cell turnover in very long-lived tissues. Optimal immunofluoresent staining results can be achieved in multiple tissue types, including pancreas, skin, gut, liver, adrenal, testis, ovary, thyroid, lymph node, and brain. We have also applied this technique to identify oncogenic transformation within tissues. We have further applied this technique to determine if transit-amplifying cells contribute to growth or renewal of tissues. In this sense, sequential administration of thymidine analogues represents a novel approach for studying the origins and survival of cells involved in tissue homeostasis.

\section{Video Link}

The video component of this article can be found at https://www.jove.com/video/2166/

\section{Protocol}

\section{Labeling Tissues with CldU and IdU}

1. Dissolve thymidine analogues (CldU or IdU) in water. Weigh $1 \mathrm{mg} / \mathrm{mL}$ of each chemical and add to separate glass bottles of distilled water We typically prepare 1 liter of each at a time.

2. Dissolve the chemicals on a stir plate or other form of agitator. CldU will dissolve in $10 \mathrm{~min}$. of agitation at room temperature. IdU requires more than an hour of agitation at $37^{\circ} \mathrm{C}$ in a shaker. Water sources can influence IdU solubility. If IdU remains unsuspended after overnight shaking, try a cleaner source of water. The solutions are stored at $4^{\circ} \mathrm{C}$ in the dark.

3. Administer the first thymidine containing solution (either CldU or IdU) to the mice for the desired labeling period. Do not allow mice to have access to unlabeled water during the period of label. When the designated labeling period is completed, begin a washout with unlabeled water for at least 24 hours (the thymidine containing analogues have serum half-lives of several hours). Administer the second thymidine containing solution (either CldU or IdU) to the mice for the desired labeling period. Refill water bottles regularly to prevent dehydration!

4. Alternatively, mice can be labeled by sequential intraperitoneal injection of CdIU and then IdU. Prepare CldU or IdU at $10 \mathrm{mg} / \mathrm{mL}$ concentration. IdU may require drop-wise addition of concentrated sodium hydroxide solution followed by vigorous shaking to go into suspension. Do not add excess sodium hydroxide. Add a single drop of sodium hydroxide to a $10 \mathrm{~mL}$ solution of IdU followed by an hour of agitation at $37^{\circ} \mathrm{C}$ in a shaker. If not in solution, repeat addition of sodium hydroxide. Inject $100 \mathrm{mcg}$ per g body weight into the intraperitoneal space.

5. Control slides are essential for this protocol. As a result, we strongly suggest investigators carry out several variations of thymidine analogue labeling to ensure sensitivity and specificity. These should include 1 . Mice that were only labeled with CldU; 2 . Mice that were only labeled with IdU; 3 . Mice that were sequentially labeled with CIdU and then IdU; 4 . Mice that were sequentially labeled in reverse order, first with IdU 
and then CldU; 5 Mice that are mock labeled with only water. When learning to label with CldU and IdU, investigators should always carry out the entire protocol with all 5 above sets of control slides from the tissue of interest.

\section{Tissue Harvest, Fixation, and Slide Preparation}

1. Using appropriate anesthetic, perform lethal overdose and then sacrifice mouse via exsanguination. Remove fresh tissue and fix with $4 \%$ paraformalehyde at $4^{\circ} \mathrm{C}$ for 24 hours. Transfer tissue to $1: 4$ formalin buffer and store at $4^{\circ} \mathrm{C}$ for embedding.

2. Alternatively, perform lethal overdose and then perfuse the mouse via the left cardiac ventricle with saline solution until blood is cleared from the body; then immediately perfuse with $30-50 \mathrm{cc}$ of $4 \%$ paraformaldehyde. Remove the tissues of interest and store in 1:4 Formalin Buffer at $4^{\circ} \mathrm{C}$ for embedding.

3. After tissue dehydration and paraffin embedding, cut 5-micron tissue sections and place onto charged slides. Bake the slides at $65^{\circ} \mathrm{C}$ for 16 hours to ensure adhesion of tissue to slide (essential with antigen retrieval steps below).

\section{Dehydration, Permeabilization, and Antigen Retrieval}

1. Remove excess paraffin by immersing slides in two xylene baths for $5 \mathrm{~min}$. each.

2. Rehydrate the tissue by immersing slides in ethanol diluted with water: $100,95,90,8070$ and $50 \%$. Soak the slides for 3 min. at each concentration, starting with $100 \%$ and going towards $50 \%$ for the final immersion.

3. Wash slides for 5 min. in $1 \times$ PBS.

4. Permeabilize cells by immersing slides in $0.2 \%$ Triton- $X$ solution for 5 min (made fresh every time).

5. Prepare slides for microwave tissue antigen retrieval. Place the slides into a glass beaker with a sufficient volume $0.01 \mathrm{M} \mathrm{pH} 6.0 \mathrm{sodium}$ citrate buffer, so that the solution covers $5 \mathrm{~cm}$ above the top of the slides to account for evaporative loss.

6. Microwave slides until the solution is boiling (3-10 min. at full power). Reduce power until solution is slowly boiling (10-80\%) and continue for an additional $20 \mathrm{~min}$. Check microwave periodically to ensure the solution remains at a slow boil.

7. Place beaker containing slides on a bench-top and allow to gently cool to room temperature, (approximately 2 hours).

8. Confirm that slides are at room temperature using a thermometer.

9. Immerse slides in $1.5 \mathrm{~N} \mathrm{HCL}$ for $40 \mathrm{~min}$. at room temperature.

10. Wash slides twice in $1 X$ PBS, 5 min. per wash.

\section{Primary and Secondary Antibodies}

1. It is important that the slides remain very moist through the entire protocol. Dry tissue will have much more auto-fluorescence, making the resulting images unusable. To prevent dying, process one slide at a time.

2. Circle each section on the slides with a liquid blocking (i.e., wax) pen. Place the slides back into the container of $1 x$ PBS.

3. Make a humid incubation chamber by placing wet paper towels laid flat on the bottom of an airtight plastic container.

4. Make up blocking solution of $10 \%$ donkey serum diluted in $1 \times$ PBS. It is important to entirely cover each section with the solution. For tissue sections that are $1 \times 1.5$ centimeters, a volume of 50 micro-liters of solution per section is sufficient.

5. Add the blocking solution to the slides. Remove slide from 1x PBS and eliminate excess liquid by gently tapping the slide edge on a stack of dry paper towels. Place slide in incubation chamber and add 50 micro-liters of $10 \%$ block solution to each section. When all the slides are completed close the container and incubate at room temperature for 1 hour.

6. Prepare primary antibody dilution to detect IdU. Dilute mouse anti-BrdU at a 1:250 concentration in $5 \%$ donkey serum made in $1 \times$ PBS Tap off block solution from slide and place back into the incubation chamber. Add the above primary antibody to each section and incubate overnight at $4^{\circ} \mathrm{C}$.

7. Prepare high stringency wash (which minimizes binding by mouse anti-BrdU antisera to CldU). Make up fresh low salt TBST buffer (36mM Tris, $50 \mathrm{mM} \mathrm{NaCl}, 0.5 \%$ tween-20; $\mathrm{pH} 8.0$ ). You will need $40 \mathrm{~mL}$ of buffer per pair of slides. Add $40 \mathrm{~mL}$ of buffer to each $50 \mathrm{~mL}$ conical tube. Before adding slides, preheat the solution to $37^{\circ} \mathrm{C}$.

8. Two at a time, remove slides from the incubation chamber, place them back-to-back (so that tissue samples face away from one another) and tap off excess primary antibody. Place the slides in the tubes filled with low salt TBST and cover. Agitate the tubes for 20 min. in a shaking bacterial culture incubator with the temperature at $37^{\circ} \mathrm{C}$, and the speed set to $225 \mathrm{rpm}$.

9. Wash the slides two times in fresh 1X PBS. 5 min. per wash.

10. Prepare the next primary antibody solution for detection of CldU and tissue antigen, if desired. Dilute rat anti-BrdU at a $1: 250$ concentration in $5 \%$ donkey serum made in $1 \times$ PBS. Dilute primary antisera against tissue antigen at working concentration into the primary antibody solution.

11. Tap excess $1 \times$ PBS off of the slides, and place them in the incubation chamber. Add primary antibody to each section and incubate overnight at $4^{\circ} \mathrm{C}$.

12. Wash the slides two times in fresh 1X PBS. 5 min. per wash.

13. Prepare the secondary antibody solution. Dilute Cy2 donkey anti-rat to 1:250 and Cy5 donkey anti-mouse to 1:500 in a $1 \mathrm{X}$ PBS solution containing $5 \%$ donkey serum. Add Cy3 secondary to detect primary species of tissue antigen antisera. Also, dilute a $0.4 \mathrm{mg} / \mathrm{mL}$ stock solution of DAPI to 1:150 in secondary antibody solution.

14. Tap excess 1 X PBS off slides and place in the incubation chamber. Add secondary antibody solution to each section and incubate for 1 hour at room temperature.

15. Wash the slides two times in fresh 1X PBS. 5 min. per wash.

16. Tap excess $1 \mathrm{X}$ PBS off of the slides and adhere cover glass with Prolong Gold anti-fade mounting media (DO NOT use Vectashield with dyanine dyes!). Press on cover slips to eliminate air bubbles. Store at $4^{\circ} \mathrm{C}$. 


\section{Imaging and Analysis}

1. Image the tissue of interest with a fluorescent microscope equipped with a high energy light source, plan-apochromatic objectives, and a high efficiency microscope camera (e.g. Hamamatsu Orca ER). Capture images in the AMCA (DAPI), Cy2 (CldU), Cy3 (Tissue antigen), and Cy5 (IdU) channels. Merge images to create a single multi-layer image file.

2. If performed correctly, DAPI stained nuclei will be homogeneously bright. Tissue antigen (e.g. insulin) should reveal cells of interest. If staining with DAPI or tissue-antigen is weak or inconsistent, immerse slide in 1X PBS overnight to remove cover slip and repeat application of secondary antisera.

3. Focus on tissue antigen in Cy3. Check CldU and IdU staining in Cy2 and Cy5 channels, toggling between channels. Look for potential crossreactivity between markers, as indicated by excessive IdU and CldU co-positivity. Look for tissues that contain only CldU or IdU. Lack of CldU or IdU positive nuclei may indicate a technical problem with labeling or staining. In this case, look for a highly replicative tissue (e.g. lymph node).

4. Analyze images. Display layers containing DAPI and tissue antigen. Using a white board marker in the dominant hand and a cell counter in the non-dominant hand, mark each nucleus with a contrasting mark with a dry erase marker (a.k.a. white board marker). Count DAPI positive cells within tissue of interest. Record total cell count. Use a dry eraser to remove marks. Display layers containing DAPI, tissue antigen, and CldU. Count CldU positive cells within tissue of interest. Record CldU cell count but do not erase marks. Change display to visualize layers containing tissue antigen, CldU, and IdU. Count CldU IdU double positive cells. Record value, erase all marks, and display layers containing DAPI, tissue antigen, and IdU. Count the total number of IdU positive cells within the tissue of interest. Record IdU cell count.

\section{Representative Results}

We sequentially labeled 6 week old female mice with CldU for one week and then IdU for two weeks, followed by immediate sacrifice. Pancreata were stained to detect CldU, IdU, and Insulin (a tissue antigen), as well as DAPI. Islets were uniformly stained with insulin. CldU stained nuclei were distinct from IdU stained nuclei (Figure 2). Many nuclei outside of the islet were CldU IdU co-positive (Figure 2, bottom right, white arrows). A single insulin positive cell had nuclear staining for both CldU and IdU (Figure 2, bottom right, magenta arrow).

Mice. All experiments with mice were performed according to the guidelines of the IACUC committee of the Children's Hospital of Philadelphia. Female B6.129 F1 wild-type mice were purchased from Taconic (Germantown New York), housed at the laboratory animal facility at The Children's Hospital of Philadelphia, and fed Mouse Diet 5015 from PMI Nutrition International (Richmond. Indiana).

Labeling strategy. By labeling the first cell division event with CldU (marked green in this scheme) and the second cell division with IdU (marked red in this scheme), sequential cell division results in co-labeled cells with both CldU and IdU (green/red).

Representative results of thymidine labeling of pancreatic cell turnover within mice. Immunofluorescent image of a mouse pancreas with islet of langerhans in the center. Mouse was infused with CldU for 1 week and then IdU for 2 weeks in the drinking water prior to immediate sacrifice. Pancreas sample was processed as described in protocol. 40x objective images, with distinct layers displayed suitable for counting. Merged images contain DAPI (white) CldU (green), IdU (red), Insulin (yellow). (upper, left) DAPI and insulin. (upper, right) CldU and Insulin. (lower, left) IdU and Insulin. (lower, right) CldU, IdU, and Insulin. Scale bar: $100 \mu \mathrm{m}$.

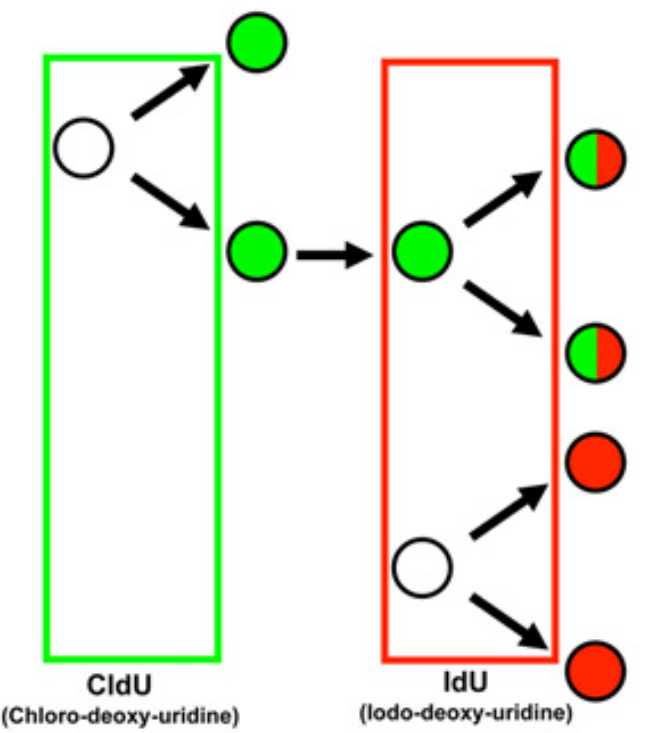

Figure 1. Labeling strategy. By labeling the first cell division event with CldU (marked green in this scheme) and the second cell division with IdU (marked red in this scheme), sequential cell division results in co-labeled cells with both CldU and IdU (green/red). 

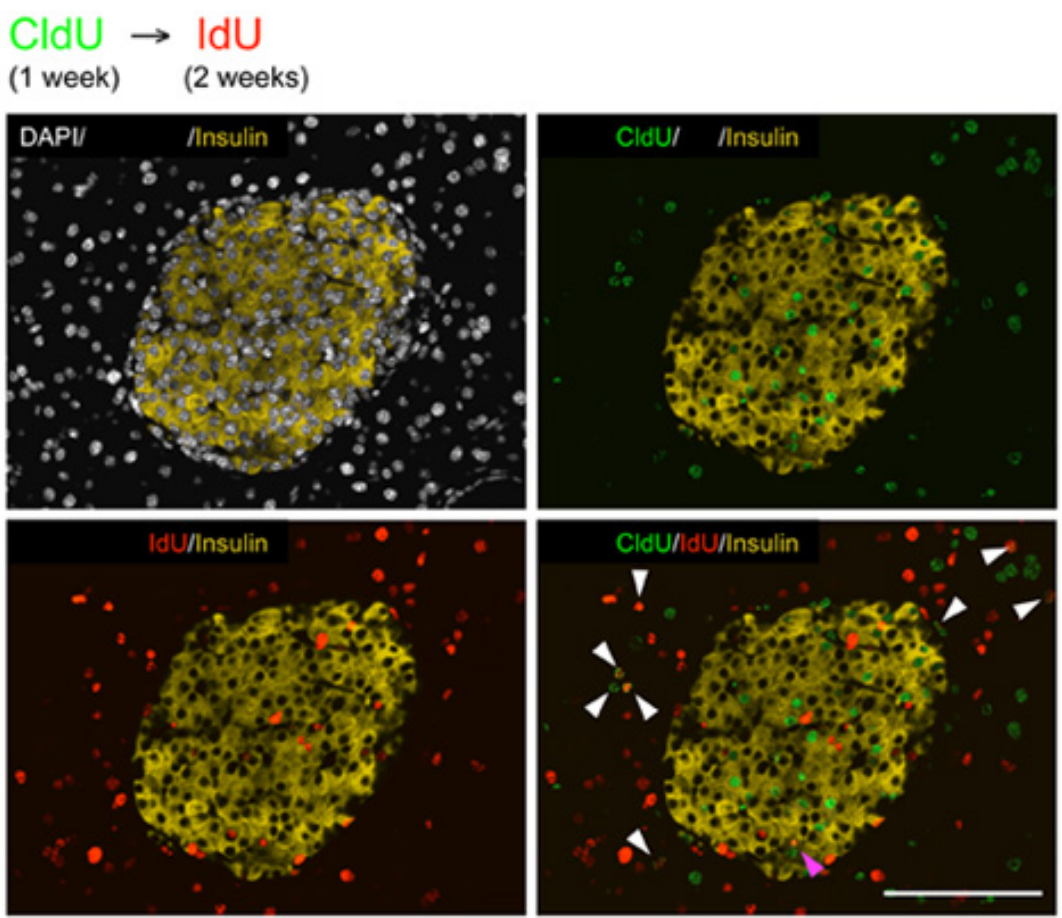

Figure 2. Representative results of thymidine labeling of pancreatic cell turnover within mice. Immunofluorescent image of a mouse pancreas with islet of langerhans in the center. Mouse was infused with CldU for 1 week and then IdU for 2 weeks in the drinking water prior to immediate sacrifice. Pancreas sample was processed as described in protocol. 40x objective images, with distinct layers displayed suitable for counting. Merged images contain DAPI (white) CldU (green), IdU (red), Insulin (yellow). (upper, left) DAPI and insulin. (upper, right) CldU and Insulin. (lower, left) IdU and Insulin. (lower, right) CldU, IdU, and Insulin. Scale bar: 100 $\mu \mathrm{m}$.

\section{Discussion}

Our approach to thymidine-based approach to labeling cell turnover has many potential applications in biomedical research. To date, we have concentrated on the pancreas, but we have also applied this strategy to examine cell turnover of skin, gut, liver, adrenal, kidney, testis, ovary, thyroid, lymph node, hematopoiesis, and brain ${ }^{1}$. Because cell turnover varies from tissue to tissue, labeling strategies should be customized to obtain the most compelling information from the organ of interest. Morrison and colleagues used CldU and IdU for 1 day each to label hematopoiesis ${ }^{2}$. Kuhn and colleagues use CldU and IdU for 4 days each to detect cell division in regenerating myocardium ${ }^{3}$. In contrast, we have used CldU and IdU for up to 9 months total to label basal cell turnover within pancreatic islets, a tissue with minimal cell turnover as the animal ages ${ }^{1,4-6}$. The most obvious potential application of our labeling strategy is for studies to define cell turnover within tissue homeostasis. But our technique has several other potential applications. For instance, we also recently applied this technique to identify oncogenic transformation within tissues, where focal areas of increased replication can be readily identified by increased incorporation of CldU or IdU ${ }^{5}$. Morrison and colleagues used CldU and IdU for 1 day each to test for label retaining behavior of hematopoietic stem cells ${ }^{2}$. We have also applied sequential thymidine analogue labeling to determine if transit-amplifying cells contribute to growth or renewal of tissues ${ }^{1}$. In this application sequential administration of thymidine analogues represents a novel approach for studying the origins and survival of cells involved in tissue homeostasis.

Thymidine analogues should not be used without caution. Synthetic thymidine analogues have potential toxicities to dividing cells, and their use could theoretically impair cell turnover in growing animals. Thymidine analogues have been used as radiosensitizing agents for cancer patients and may slow cell turnover. Thus we urge investigators to cautiously investigate for potential toxicities in their tissue of interest. For example, Trumpp and colleagues find that systemic BrdU can force hematopoietic stem cells to enter cell cycle ${ }^{7}$. For instance, Rutter and colleagues observed that high dose thymidine analogues are toxic to developing pancreas ${ }^{8}$. More recently Hellerstein and colleagues at KineMed, Inc found that $\mathrm{BrdU}$ administration slows intra-islet proliferation by $16-25 \%$ using a proprietary heavy water labeling technique ${ }^{9}$. The whole islet preparations used by Hellerstein and colleagues contained many other endocrine and non-endocrine cell types, which could comprise as much as $50 \%$ of total islet preparations. However, we find that prolonged infusion of BrdU does not influence beta-cell proliferation in young adult mice, as measured by ki67 expression ${ }^{4}$. Similarly, long-term administration of CldU and IdU does not seem to impair beta cell mass expansion ${ }^{1}$. Moreover, beta cell proliferation rates are equivalent in between mice labeled with BrdU for long periods of time and mice that are only labeled for a few hours (rates are normalized to equivalent time periods). Together, these results suggest that mice can safely tolerate long-term lowdose administration of thymidine analogues. Still, we cannot rule out potential toxicity by thymidine analogues in pancreatic beta cell growth. As a result, we continue to perform controls when labeling mice with thymidine analogues, and urge other investigators to do so as well.

Our technique of sequential thymidine analogue labeling is not without pitfalls and technical challenges. As a result, control slides are especially important. We have generated control slides that consist of various tissues from 1.) Mice that were only labeled with CldU; 2.) Mice that were only labeled with IdU; 3.) Mice that were sequentially labeled with CldU and then IdU; 4.) Mice that were sequentially labeled in reverse order, first with IdU and then CldU; 5.) Mice that are mock labeled with only water. When learning to label with CldU and IdU, investigators should always carry out the entire protocol with all 5 above sets of slides from the tissue of interest. 
Slight cross reactivity in between CldU and IdU is an unfortunate but unavoidable downside to labeling with both thymidine analogues. The CldU and IdU technique is based on differences in affinities in between antisera that were originally derived against BrdU in different species (mouse and rat). Our protocol, while cumbersome, is designed to minimize such cross reactivity. Consequently, we urge caution amongst investigators who consider skipping step(s) of the protocol. In particular, we have encountered problems with secondary antisera that are cross reactive in between mouse and rat. This can be minimized by the use of Jackson antisera that are fully cross-adsorbed in between mouse and rat.

We occasionally fail to adequately detect thymidine incorporation. In such instances it is critically important to use positive control slides to determine which reagent or step was not working. Difficulties are typically due to bad aliquots of antisera, dry slides, or inadequate nuclear permeabilization. We have failed to successfully label developing embryos with IdU. Thus, not all tissues may be permeable to IdU.

Alternatives to CldU and IdU for double thymidine analogue labeling are on the horizon. EdU (5-ethinyl-2 deoxyuridine) can be a substrate for copper catalyzed click chemistry detection ${ }^{10,11}$. EdU detection methods do not require separation of DNA strands, and are thus highly amenable to analysis by flow cytometry ${ }^{12}$. EdU is compatible but not cross reactive with BrdU ${ }^{10}$. EdU has been used to quantify cell turnover of various mouse tissues including pancreatic beta cells ${ }^{13}$, intestinal L cells ${ }^{14}$ and Epidermis ${ }^{15}$. EdU is fairly expensive at the moment $(\$ 1000$ US per $500 \mathrm{mg}$ ). Still, Edu detection appears to be much more sensitive than BrdU detection ${ }^{10}$. Thus, it might be economically feasible to combine EdU and BrdU instead of CldU and IdU. This method might also allow detection of three different rounds of cell division in mice triply labeled for EdU, CldU, and IdU.

In summary, our technique of sequential thymidine analogue labeling is a novel approach to detecting cell turnover. We hope our approach enables other scientists to more accurately quantify cell division, and expect that it will open new paradigms in tissue homeostasis.

\section{Disclosures}

All experiments with mice were performed in the animal facility at The Children's Hospital of Philadelphia according to the guidelines of the Institutional Animal Care and Use Committee (IACUC).

\section{Acknowledgements}

Supported by the JDRF, the NIH (R01-DK081469), the Commonwealth of Pennsylvania (Center for Excellence in Regenerative Medicine grant 4100043362), the March of Dimes (Basil O'Connor Starter Scholar Research Award), and a University of Pennsylvania DERC pilot and feasibility grant (DK19525).

\section{References}

1. Teta, M. et al., Growth and regeneration of adult Beta cells does not involve specialized progenitors. Dev Cell 12 (5), 817 (2007).

2. Kiel, M. J. et al., Haematopoietic stem cells do not asymmetrically segregate chromosomes or retain BrdU. Nature 449 (7159), 238 (2007).

3. Bersell, K., Arab, S., Haring, B., and Kuhn, B., Neuregulin1/ErbB4 signaling induces cardiomyocyte proliferation and repair of heart injury. Cell 138 (2), 257 (2009).

4. Rankin, M. M. and Kushner, J. A., Adaptive Beta Cell Proliferation Is Severely Restricted with Advanced Age. Diabetes (2009).

5. He, L. M. et al., Cyclin D2 Protein Stability Is Regulated in Pancreatic Beta Cells. Mol Endocrinol (2009).

6. Kushner, J. A., Beta-cell growth: an unusual paradigm of organogenesis that is cyclin D2/Cdk4 dependent. Cell Cycle 5 (3), 234 (2006); Teta, M. et al., Very slow turnover of beta-cells in aged adult mice. Diabetes 54 (9), 2557 (2005).

7. Wilson, A. et al., Hematopoietic stem cells reversibly switch from dormancy to self-renewal during homeostasis and repair. Cell 135 (6), 1118 (2008).

8. Van Nest, G., Raman, R. K., and Rutter, W. J., Effects of dexamethasone and 5-bromodeoxyuridine on protein synthesis and secretion during in vitro pancreatic development. Dev Biol 98 (2), 295 (1983); Githens, S., Pictet, R., Phelps, P., and Rutter, W. J., 5-bromodeoxyuridine may alter the differentiative program of the embryonic pancreas. J Cell Biol 71 (2), 341 (1976); Walther, B. T., Pictet, R. L., David, J. D., and Rutter, W. J., On the mechanism of 5-bromodeoxyuridine inhibition of exocrine pancreas differentiation. J Biol Chem 249 (6), 1953 (1974).

9. Chen, S. et al., Measurement of pancreatic islet cell proliferation by heavy water labeling. Am J Physiol Endocrinol Metab 293 (5), E1459 (2007).

10. Salic, A. and Mitchison, T. J., A chemical method for fast and sensitive detection of DNA synthesis in vivo. Proc Natl Acad Sci U S A 105 (7), 2415 (2008).

11. Best, M. D., Click chemistry and bioorthogonal reactions: unprecedented selectivity in the labeling of biological molecules. Biochemistry 48 (28), 6571 (2009).

12. Buck, S. B. et al., Detection of S-phase cell cycle progression using 5-ethynyl-2'-deoxyuridine incorporation with click chemistry, an alternative to using 5-bromo-2'-deoxyuridine antibodies. Biotechniques 44 (7), 927 (2008).

13. Huising, M. O. et al., CRFR1 is expressed on pancreatic beta cells, promotes beta cell proliferation, and potentiates insulin secretion in a glucose-dependent manner. Proc Natl Acad Sci U S A 107 (2), 912.

14. Reimann, F. et al., Glucose sensing in L cells: a primary cell study. Cell Metab 8 (6), 532 (2008).

15. Doupe, D. P., Klein, A. M., Simons, B. D., and Jones, P. H., The ordered architecture of murine ear epidermis is maintained by progenitor cells with random fate. Dev Cell 18 (2), 317. 